\title{
ASSOCIAÇÃO DE FORÇA E NÍVEL DE ATIVIDADE FÍSICA À DENSIDADE MINERAL ÓSSEA NA PÓS-MENOPAUSA
}

\author{
ASSOCIATION OF FORCE AND PHYSICAL ACTIVITY LEVEL WITH BONE MINERAL \\ DENSITY IN POSTMENOPAUSE
}

Artigo Original

ORIGINAL ARTICLE

Artículo OrIGINAL

\author{
ASOCIACIÓN DE FUERZA Y NIVEL DE ACTIVIDAD FÍSICA A LA DENSIDAD MINERAL \\ ÓSEA EN LA POSTMENOPAUSIA
}

\author{
Cristiane Fialho Ferreira da Silva ${ }^{1}$ \\ (Fisioterapeuta) \\ Paulo Roberto dos Santos Amorim \\ (Educador Físico) \\ Cristiane Junqueira de Carvalho \\ (Médica Reumatologista) \\ Mariana Maia de Faria' \\ (Graduanda em Medicina) \\ Luciana Moreira Lima' \\ (Bioquímica) \\ 1. Universidade Federal de Viçosa, \\ Viçosa, MG, Brasil.

\section{Correspondência: \\ Luciana Moreira Lima. Universidade Federal de Viçosa, Departamento de Medicina e Enfermagem. \\ Av. PH Rolfs, s/n - Centro. Viçosa, MG, Brasil. 36570-000. \\ luciana.lima@ufv.br}

\section{RESUMO}

Introdução: o exercício físico atua melhorando a densidade mineral óssea (DMO) por gerar deformidades nesse tecido e estimular remodelação. Objetivo: verificar a associação entre força muscular e nível de atividade física à densidade mineral óssea (DMO) utilizando testes de força de bíceps, de sentar e levantar, dinamometria de mãos, história pregressa de atividade física e nível de atividade física, avaliado pelo pedômetro com a DMO em mulheres na pós-menopausa. Métodos: estudo transversal, descritivo que mensurou a densidade mineral óssea pela absorção de dupla energia de raios X (DXA) da coluna lombar (L1-L4), fêmur e antebraços em 62 mulheres saudáveis no pós-menopausa, com média de 56,82 \pm 4,02 anos de idade. Foi aplicado questionário para atividade física pregressa e realizada contagem diária de passos (pedômetro). A força muscular foi medida pelos testes de dinamometria de mãos, de 30 segundos de bíceps bilateral e de sentar e levantar da cadeira em 30 segundos. Realizou-se registro alimentar de três dias para mensuração da ingestão diária de cálcio e vitamina D. Resultados: os indivíduos apresentaram altos níveis de atividade física, porém baixa ingestão diária de cálcio e vitamina D. Não foi verificada diferença estatisticamente significativa entre força muscular nos dois grupos estudados, com DMO diminuída e DMO normal. O grupo com DMO diminuída apresentou maior número de passos diários e menor peso quando comparado com o grupo com DMO normal. Foram observadas várias correlações positivas de baixa magnitude, embora significantes, entre a densitometria e a força muscular ( $p<0,50$ ). Conclusão: os autores descreveram risco cinco vezes e meia maior em mulheres não praticantes de atividade física da adolescência até a idade adulta e de apresentarem redução da DMO em comparação com as mulheres que apresentaram DMO normal.

Palavras-chave: exercício, músculos, densidade óssea, doenças ósseas metabólicas.

\section{ABSTRACT}

Introduction: physical activity acts improving bone mineral density (BMD) because it generates deformities in the tissue and stimulates remodeling. Objective: to determine the association between muscle strength and level of physical activity with bone mineral density (BMD) using biceps strength tests, sitting and standing, hands grip strength, history of physical activity and physical activity level, evaluated by pedometer with BMD in postmenopausal women. Methods: cross-sectional, descriptive study that measured the bone mineral density by dual energy absorption of $X$ rays (DXA) of the lumbar spine (L1-L4), femur and forearm in 62 health postmenopausal women, with average age of $56.82 \pm 4.02$ years. A questionnaire was applied to assess previous physical activity and a daily step count (pedometer) was performed. Muscle strength was measured by grip strength tests, 30 seconds of bilateral biceps and sitting and standing test in 30 seconds. A 3-day food registering was performed to measure the daily intake of calcium and vitamin D. Results: participants had high levels of physical activity, but low daily intake of calcium and vitamin $D$. There was no statistically significant difference in muscle strength in both groups, with decreased BMD and normal BMD. The group with decreased BMD showed a higher number of daily steps and less weight when compared with the group with normal BMD. Several positive correlations of low magnitude although significant were observed between densitometry and muscle strength $(p<0.50)$. Conclusion: the authors related risk five and half times higher in women not engaged in physical activity from adolescence to adulthood, and decreased BMD compared with women with normal BMD.

Keywords: exercise, muscles, bone density, bone diseases, metabolic.

\section{RESUMEN}

Introducción: Ios ejercicios físicos actúan mejorando la densidad mineral ósea (DMO) por generar deformidades en ese tejido y estimular remodelación. Objetivo: verificar la asociación entre fuerza muscular y nivel de actividad física a la densidad mineral ósea (DMO) utilizando tests de fuerza de bíceps, de sentarse y levantarse, dinamometría de manos, historia anterior de actividad fisica y nivel de actividad física, evaluado por el podómetro con la DMO en mujeres en la postmenopausia. Métodos: estudio transversal, descriptivo que midió la densidad mineral ósea por la absorción de doble energía de rayos X (DXA) de la columna lumbar (L1-L4), fémur y antebrazos en 62 mujeres 
saludables en la postmenopausia, con promedio de 56,82 \pm 4,02 años de edad. Fue aplicado cuestionario para actividad física anterior y realizado conteo diario de pasos (podómetro). La fuerza muscular fue medida por los tests de dinamometría de manos, de 30 segundos de bíceps bilateral y de sentarse y levantarse de la silla en 30 segundos. Se realizó registro alimentario de tres días para medición de la ingestión diaria de calcio y vitamina D. Resultados: los individuos presentaron altos niveles de actividad física, aunque baja ingestión diaria de calcio y vitamina D. No fue verificada diferencia estadísticamente significativa entre fuerza muscular en los dos grupos estudiados, con DMO disminuida y DMO normal. El grupo con DMO disminuida presentó mayor número de pasos diarios y menor peso cuando comparado con el grupo con DMO normal. Fueron observadas varias correlaciones positivas de baja magnitud, aunque significativas, entre la densitometría y la fuerza muscular $(p<0,50)$. Conclusión: los autores describieron riesgo cinco veces y media mayor en mujeres no practicantes de actividad física de la adolescencia hasta la edad adulta y de presentar reducción de la DMO en comparación con las mujeres que presentaron DMO normal.

Palabras clave: ejercicio, músculos, densidad ósea, enfermedades óseas metabólicas.

\section{INTRODUÇÃO}

Exercício físico atua melhorando a densidade mineral óssea (DMO) por gerar deformidades nesse tecido e estimular remodelação', sendo os de alto impacto ou que exijam grande produção de força os mais correlacionados a esses benefícios ${ }^{1-3}$. Porém, existem controvérsias quanto à relação da DMO com força muscular e nível de atividade física ou quais desses fatores estariam mais associados à sua melhora e manutenção ${ }^{2-5}$. Outra questão seria a duração, frequência e tipos de atividades, visto que exercícios habituais podem não ser efetivos para o anabolismo ósseo e se excessivos poderiam prejudicar a homeostasia tecidual ${ }^{3}$.

Embora não haja consenso na literatura, indivíduos com maior força muscular parecem apresentar maiores taxas relativas a DMO, uma vez que a contração muscular é mecanismo de sobrecarga óssea ${ }^{3,5}$. Pruitt et al. apud Cadore et al. ${ }^{2}$ sugeriram que o estímulo à deformação óssea pode ocorrer quando a contração muscular ultrapassa determinado limiar de esforço, sendo necessários treinamentos intensos para aprimoramento da $\mathrm{DMO}^{2}$. Kemmler et al. ${ }^{3}$ não observaram associação do nível de atividade física e força isométrica máxima com DMO em mulheres na pós-menopausa precoce; contudo, através de análise de regressão força de braços explicou quatro e meio porcento da variação da DMO em antebraços ${ }^{3}$.

Testes de força e equilíbrio são meios seguros, práticos e com alta confiabilidade para avaliação de mulheres na pós-menopausa. Teste de sentar e levantar de 30 segundos tem sido utilizado para avaliar risco de quedas e fraturas 6 . Testes de bíceps de 30 segundos e dinamometria de mão proporcionam avaliação do estado geral de saúde e apresentam correlação com DMO em alguns sítios ósseos. Costa et al. ${ }^{7}$ observaram correlação da força manual com DMO do colo do fêmur $(r=0,582, p=0,003)$ e do fêmur total $(r=0,485, p=0,01)$.

O nível de atividade física tem sido ainda correlacionado a $\mathrm{DMO}^{8,9}$, porém estes estudos são escassos. O pedômetro, método simples, de baixo custo, boa confiabilidade e validade, tem sido usado para correlacionar o nível de atividade física com parâmetros antropométricos e clínicos em populações de idade variável ${ }^{10-12}$. Estudos têm relacionado a intensidade e número de passos necessários para aprimoramento da massa óssea. Boyer et al. ${ }^{13}$ demonstraram que indivíduos com menor peso necessitavam de mais que 10000 passos diários para obtenção de benefícios. Tudor-Loker e Bassett ${ }^{11}$ relataram que não existem evidências de que o excesso de atividade seria associada a prejuízos à saúde. No entanto, Kemmler et al. ${ }^{3}$ não encontraram associação entre nível de atividade física e $\mathrm{DMO}$, observando que outros estudos apresentavam $r<0,3$, negligenciando fatores de confusão como idade e dieta.
O objetivo desse estudo foi verificar relação dos testes de força de bíceps, sentar e levantar, dinamometria de mãos, história de atividade física pregressa e nível de atividade física habitual com DMO em mulheres na pós-menopausa.

\section{CASUISTICA E MÉTODO}

Após aprovação pelo Comitê de Ética em Pesquisa com Seres Humanos da Universidade Federal de Viçosa (UFV), protocolo n ${ }^{0} 431.676$, realizou-se estudo transversal, observacional e descritivo.

As participantes foram selecionadas no Município de Viçosa, Minas Gerais, tendo como critérios de inclusão: idade entre 50 e 70 anos; status pós-menopausa; ausência de histerectomia prévia, diabetes, distúrbios de tireoide e reposição hormonal por ao menos um ano.

Explicados os objetivos e etapas, e, após assinatura do Termo de Consentimento Livre e Esclarecido, formulado segundo normas da resolução 466/2012 do Ministério da Saúde (MS) para pesquisa com seres humanos, as voluntárias passaram por exame com médico clínico geral/reumatologista. Foram critérios de exclusão: uso de medicamentos que afetem metabolismo ósseo, fraturas recentes (menos de um ano), dificuldades de deambulação, história de hipertensão ou insuficiência cardíaca grave e cirurgia bariátrica. Avaliaram-se 95 voluntárias: 24 casos foram excluídos. Entre os 71 casos selecionados, ocorreram sete desistências espontâneas e dois casos sofreram fraturas, restando para a casuística 62 indivíduos.

Na primeira etapa, realizou-se registro alimentar de três dias ${ }^{14}$. Na segunda, avaliação física, testes de força, aplicação de questionário de atividade física pregressa que leva em consideração a sobrecarga óssea das atividades - bone loading history questionnaire $(B L H Q)^{15}$ e colocação do pedômetro. O questionário de sobrecarga óssea - bone loading history questionnaire - que proporciona escore de sobrecarga na coluna lombar e fêmur proximal direito, foi utilizado para avaliar as voluntárias como praticantes ou não de atividade física com sobrecarga óssea de intensidade moderada a alta na infância, adolescência e idade adulta. Para cálculo de índice de massa corporal (IMC), todas foram pesadas e medidas apenas com roupa íntima em balança mecânica antropométrica (WELMY/ Brasil) com precisão de $100 \mathrm{~g}$ e $0,5 \mathrm{~cm}$. Os testes de força executados foram:

1) Dinamometria de mãos: utilizou-se dinamômetro modelo JAMAR ${ }^{\circledR}$ - Sammons Preston, INC Bolingbrook, IL 60440, EUA. Sentadas, cotovelo fletido a $90^{\circ}$ e antebraço em posição neutra ${ }^{16}$, as voluntárias realizaram três repetições de cinco segundos de força máxima, alternadamente, com intervalo de um minuto. Considerou-se a medida de maior valor registrada em $\mathrm{kg} / \mathrm{f}^{17}$. 
2) Teste de 30 segundos de bíceps: utilizou-se halter de dois quilos e foram realizados o máximo de flexões e extensões em cada membro, separadamente, na posição sentada, com intervalo de um minuto de descanso ${ }^{18}$. Foram registrados apenas movimentos de flexão/ extensão completos. 3) Teste de sentar e levantar em 30 segundos: sentada em cadeira sem braços, tronco apoiado no encosto, pés no chão e braços cruzados sob tórax. Registrou-se o maior número de vezes que se levantavam e retornavam a sentar ${ }^{18}$.

Ao final, todas foram orientadas a utilizar o pedômetro (DIGI-WALKER modelo SW200, Yammax, Japão) por sete dias, colocando-o à direita na cintura em direção à linha média do joelho, sendo permitida retirada para dormir, banho e atividades aquáticas. A partir da média de passos diários, classificou-se como: "muito ativas" com média maior que 12500 passos, "ativas" entre 10000 e 12499, "insuficientemente ativas" de 5000 a 9999 e "sedentárias" quando abaixo de 5000"19,20.

Na terceira etapa, as voluntárias compareciam à Divisão de Saúde (DSA) da UFV em jejum de pelo menos oito horas, entre as sete e nove da manhã, para exame de densitometria óssea. Medidas foram obtidas pelo método de absorção de dupla energia de raios-X (DXA), utilizando o aparelho Lunar Prodigy Advance DXA System (analysis version: 13,31) fabricado por GE Medical, model 8743, Madison, WI, USA. Foram verificadas as DMO da coluna lombar ( $L 1-L 4)$, fêmures proximais totais e colos femorais, antebraços distais e corpo total. Para análise dos dados as voluntárias foram divididas em dois grupos (I- controle: DMO normal e II - baixa DMO) nos sítios da coluna lombar (L1-L4), fêmur proximal total e colo, corpo total, antebraços distais e combinação do sítio lombar com fêmur proximal (denominada DMO total).

As classificações seguiram definições da Organização Mundial de Saúde (1994), utilizando o T score. Foram consideradas duas categorias: DMO normal (T score $\geq-1$ ) e diminuída $(T \text { score }<-1)^{21}$.

\section{Análise estatística}

Os resultados estão descritos em médias e desvio padrão. Através do Teste de Kolmogorov-Smirnov pode-se verificar distribuição normal das variáveis passos diários e testes de força. Nessas, utilizou-se Teste $t$ de Student para grupos independentes comparando médias da densitometria óssea. Teste de Odds Ratio avaliou força de associação entre história pregressa de atividade física na infância até idade adulta em relação à DMO total.

Os testes de Pearson e Spearman avaliaram as correlações entre variáveis contínuas e dicotômicas, respectivamente. Qui-quadrado e Exato de Fisher analisaram as variáveis categóricas.

Utilizou-se programa estatístico IBM SPSS versão 20 e nível de significância $p \leq 0,05$.

\section{RESULTADOS}

Participaram do estudo 62 mulheres na pós-menopausa (Tabela 1) com média de idade de 56,82 \pm 4,02, sendo que: 25 (40,3\%) tinham de 50 a 55 anos, $27(43,5 \%)$ de 56 a 60 e $10(16,1 \%)$ entre 61 e 66 . A média do IMC foi de 27,52; observou-se que 25 mulheres (40,3\%) apresentaram IMC normal, $22(35,5 \%)$ estavam com sobrepeso e $15(24,2 \%)$ eram obesas. $O$ grupo com DMO normal apresentou maiores valores de IMC e peso, sendo a diferença estatisticamente significativa em relação ao outro grupo. Os registros alimentares demonstraram baixa ingestão de cálcio e vitamina $D$ em toda a amostra, com média de 448,32 $\pm 226,68 \mathrm{mg} /$ dia de cálcio e 1,02 $\pm 0,94 \mathrm{mcg} /$ dia de vitamina $\mathrm{D}$, sem diferenças estatísticas entre os grupos.

Houve dependência pelo teste de Qui-quadrado da suplementação de cálcio $\left(x^{2}=0,025\right)$ e suplementação combinada de cálcio e vitamina $D\left(x^{2}=0,048\right)$ com a classificação pelo $T$ score, porém houve independência em relação à suplementação somente com vitamina $\mathrm{D}\left(\mathrm{x}^{2}=0,539\right)$.
Na tabela 2 estão demonstrados os valores médios dos passos medidos pelo pedômetro por sete dias totais (uma semana completa), seis (retirou-se o primeiro dia para evitar efeito Hawthorne), cinco (dias da semana), quatro (três dias de semana e um de fim de semana), três (dois dias de semana e um de fim de semana) e dois dias de fim de semana.

Tabela 1. Características gerais da amostra e diferenças estatísticas em relação à classificação pelo T score (coluna lombar e fêmur proximal direito).

\begin{tabular}{|c|c|c|c|c|}
\hline Características & $\begin{array}{c}\text { Amostra } \\
\text { Total }\end{array}$ & $\begin{array}{l}\text { DMO } \\
\text { Normal }\end{array}$ & $\begin{array}{c}\text { DMO } \\
\text { diminuída }\end{array}$ & $p$ \\
\hline Idade & $n=62$ & $n=22$ & $n=40$ & $0,063^{*}$ \\
\hline $50-55$ anos & $56,82 \pm 4,02$ & $55,55 \pm 4,10$ & $57,53 \pm 3,85$ & $0,005^{* *}$ \\
\hline $56-60$ anos & $25(40,3 \%)$ & $14(63,6 \%)$ & $11(27,5 \%)$ & $0,017^{* *}$ \\
\hline $61-66$ anos & $27(43,5 \%)$ & $5(22,7 \%)$ & $22(55 \%)$ & $0,320^{* * *}$ \\
\hline Estatura & $10(16,1 \%)$ & $3(13,6 \%)$ & $7(17,5 \%)$ & $0,753^{*}$ \\
\hline Peso & $1,54 \pm 0,07$ & $1,55 \pm 0,06$ & $1,54 \pm 0,07$ & $0,149^{*}$ \\
\hline IMC & $65,45 \pm 9,73$ & $67,86 \pm 9,37$ & $64,12 \pm 9,79$ & $0,171^{*}$ \\
\hline Normal & $27,52 \pm 3,87$ & $28,43 \pm 3,97$ & $27,02 \pm 3,78$ & $0,036^{* *}$ \\
\hline Sobrepeso & $25(40,3 \%)$ & $5(22,7 \%)$ & $20(50 \%)$ & $0,046^{* *}$ \\
\hline Obesidade & $22(35,5 \%)$ & $12(54,5 \%)$ & $10(25 \%)$ & $0,549 * *$ \\
\hline Fósforo (mg/dL) & $15(24,2 \%)$ & $5(22,7 \%)$ & $10(25 \%)$ & $0,294 * * * *$ \\
\hline $\begin{array}{l}\text { Cálcio iônico } \\
\text { (mg/dL) }\end{array}$ & $\begin{array}{c}3,80 \\
(3,58-4,10)\end{array}$ & $3,8(3,40-4,03)$ & $3,8(3,7-4,10)$ & $0,467^{*}$ \\
\hline PTH (pg/mL) & $4,76 \pm 0,16$ & $4,74 \pm 0,12$ & $4,77 \pm 0,18$ & $0,902^{*}$ \\
\hline Cálcio na dieta & $38,27 \pm 14,55$ & $38,59 \pm 14,75$ & $38,10 \pm 14,63$ & $0,682^{*}$ \\
\hline Vitamina D na dieta & $448,32 \pm 226,68$ & $464,44 \pm 246,93$ & $439,46 \pm 217,51$ & $0,314^{*}$ \\
\hline $\begin{array}{l}\text { Suplementação } \\
\text { com cálcio }\end{array}$ & $1,02 \pm 0,94$ & $1,04 \pm 0,71$ & $1,30 \pm 1,04$ & \\
\hline Sim & & & & --- \\
\hline Não & $8(12,9 \%)$ & 0 & $8(20 \%)$ & --- \\
\hline $\begin{array}{l}\text { Suplementação } \\
\text { com Vitamina D }\end{array}$ & $54(87,1 \%)$ & $22(100 \%)$ & $32(80 \%)$ & \\
\hline Sim & & & & $0,176^{* * *}$ \\
\hline \multirow[t]{2}{*}{ Não } & $14(22,58 \%)$ & $3(13,6 \%)$ & $11(27,5 \%)$ & $0,537^{* *}$ \\
\hline & $48(77,42 \%)$ & $17(86,4 \%)$ & $28(72,5 \%)$ & \\
\hline
\end{tabular}

*Teste de Student, ${ }^{* *}$ Teste de Qui-quadrado, ${ }^{* * *}$ Teste Exato de Fisher, ${ }^{* * * *}$ Teste de Mann-Whitney, $\mathrm{p}=$ probabilidade.

Tabela 2. Diferença entre os grupos de DMO da lombar em relação à média da contagem diária de passos (pedômetro) em intervalos diferentes de dias de uma semana.

\begin{tabular}{c|c|c|c}
\hline Variáveis & $\begin{array}{c}\text { DMO Lombar Diminuída } \\
\mathbf{n = 3 8}\end{array}$ & $\begin{array}{c}\text { DMO Lombar Normal } \\
\mathbf{n}=\mathbf{2 4}\end{array}$ & $\boldsymbol{p}$ \\
\hline MD (7 dias) & $9475,26 \pm 3792,27$ & $7912,64 \pm 2800,52$ & 0,087 \\
\hline MD (6 dias) & $9340,23 \pm 3731,98$ & $7868,8 \pm 2892,92$ & 0,106 \\
\hline MD (5 dias) & $10085,6 \pm 4247,86$ & $8129,73 \pm 2766,85$ & $0,050^{*}$ \\
\hline MD (4 dias) & $9197,35 \pm 3658,18$ & $7795,86 \pm 3048,37$ & 0,123 \\
\hline MD (3 dias) & $9907,07 \pm 4002$ & $7607,46 \pm 2836,72$ & $0,017^{*}$ \\
\hline MD (2 dias, FDS) & $7897,2 \pm 4075,28$ & $7369,63 \pm 4206,56$ & 0,626 \\
\hline
\end{tabular}

$\mathrm{p}=$ probabilidade para o teste de hipótese (Teste $T$ de Student), $\mathrm{DMO}=$ densidade mineral óssea, $\mathrm{MD}=$ médi de passos por intervalo de dias; FDS = fim de semana. 
Houve diferença estatisticamente significativa do número de passos entre os grupos na coluna vertebral ao se considerar o pedômetro em cinco e três dias, constatando que o grupo com DMO diminuída apresentou maior número de passos. Porém, não foi observada diferença estatisticamente significativa em relação ao fêmur proximal total e colo direito nas cinco categorias de uso do pedômetro.

Quanto aos testes de força, não houve diferenças significativas entre as variáveis nos dois grupos estudados na coluna lombar, fêmur proximal total, colo direito e antebraço terço distal direito.

A tabela 3 apresenta as correlações positivas e significativas observadas entre a densitometria e força.

A tabela 4 mostra valor de cinco e meio maior chance de ocorrer DMO diminuída na ausência de atividade física de sobrecarga óssea de moderada à alta com nível de significância de 0,01.

Tabela 3. Correlação de Pearson entre as medidas de densitometria e força muscular.

\begin{tabular}{c|c|c}
\hline & $\begin{array}{c}\text { Força de preensão palmar direita } \\
\text { (coeficiente de correlação) }\end{array}$ & $\boldsymbol{p}$ \\
\hline $\begin{array}{c}\text { DXA antebraço direito } \\
\text { distal total }\end{array}$ & $0,377^{* *}$ & 0,008 \\
\hline $\begin{array}{c}\text { DXA antebraço direito } \\
\text { distal distal }\end{array}$ & $0,311^{*}$ & 0,031 \\
\hline $\begin{array}{c}\text { DXA antebraço esquerdo } \\
\text { distal total }\end{array}$ & $0,335^{*}$ & 0,020 \\
\hline $\begin{array}{c}\text { DXA antebraço esquerdo } \\
\text { distal distal }\end{array}$ & $0,346^{*}$ & 0,016 \\
\hline DXA corpo total & $0,430^{* *}$ & 0,002 \\
\hline
\end{tabular}

** Correlação é significativa ao nível de 0,01 ; * Correlação é significativa ao nível de $0,05 . D X A=$ absorção de dupla energia de raios $X, p=$ probabilidade.

Tabela 4. Razão de chance de ocorrer DMO diminuída em relação ao histórico de atividade física.

\begin{tabular}{c|c|c|c}
\hline Atividade Física Pregressa & DMO total Diminuída & DMO total Normal & $\boldsymbol{p}$ \\
\hline N & 40 & 22 & \\
\hline Sim & $20(50 \%)$ & $20(90,9 \%)$ & \\
\hline Não & $20(50 \%)$ & $2(9,1 \%)$ & \\
\hline Odds Ratio & 5,500 & 0,550 & 0,002 \\
\hline
\end{tabular}

$\mathrm{DMO}=$ Densidade Mineral Óssea, $p=$ probabilidade, $\mathrm{n}=$ número de individuos.

\section{DISCUSSÃO}

Os principais fatores de risco para o desenvolvimento de osteoporose e fraturas são o sexo feminino e baixa $\mathrm{DMO}^{22,23}$, sendo de grande importância a detecção precoce da diminuição da DMO nessa população. Como a densitometria óssea ainda é método dispendioso, medidas indiretas podem contribuir para triagem de indivíduos em risco. O presente estudo testou a relação de força e nível de atividade física com a DMO de mulheres na pós-menopausa. Não foram verificadas diferenças estatisticamente significativas de força muscular nos grupos avaliados. Apesar de ter sido demonstrada correlações positivas da dinamometria de mãos com os valores de T-score nos sítios ósseos do antebraço direito distal total, distal-distal e corporal total, essas correlações foram baixas. Os resultados encontrados corroboram com o estudo de Kemmler et al. ${ }^{3}$ que não encontrou associação entre força e nível de atividade física habitual com a DMO. Marin et al. ${ }^{24}$ demonstrou correlação moderada de força de mão com DMO.

Em nosso estudo, pedômetro por três dias apresentou diferença estatisticamente significativa entre os dois grupos, sendo que o com
DMO diminuída apresentou maior número de passos diários. Evidenciando que número de passos pode não ser o principal estímulo para remodelação óssea na coluna lombar, podendo sofrer interferência de outros fatores como genética, homeostase hormonal e nutrição 2,9 . Não foram encontradas correlações dos valores da DMO no sítio da coluna com valores obtidos pelo pedômetro. Porém estudos apontam que a remodelação óssea necessita de atividades com maior sobrecarga e forças de reação do solo ${ }^{25-27}$, melhores mensuradas pelo acelerômetro ${ }^{11}$.

No presente estudo não houve mulheres classificadas como sedentárias, não sendo possível comparação com o grupo ativo. No entanto, número de passos menor que 5000 tem sido associado às doenças crônicas e demais alterações de saúde ${ }^{11}$. Cabe ressaltar que a presença de apenas mulheres saudáveis na pós-menopausa em nosso estudo pode ter interferido nos resultados. Estudos relatam que 10000 passos seriam necessários para classificar o indivíduo como ativo 19,20 com equivalência de gasto calórico em torno de 300 a $400 \mathrm{Kcal}$ por dia, dependendo do peso do indivíduo e velocidade dos passos. Recomendações de atividade física para saúde indicam ao menos 30 minutos diários com intensidade moderada, que equivaleria de 3000 a 4000 passos $^{11}$. Porém, a rotina diária de trabalhos domésticos de muitas mulheres fica em torno 6000 passos e acrescido da caminhada de 30 minutos poderia então alcançar valores de 10000 passos preconizados como ideal para melhorias na saúde - diminuição de peso, controle da glicemia, colesterol, hipertensão e possível ganho na $\mathrm{DMO}^{11,27}$.

Analisando o peso das nossas mulheres, pode-se constatar que aquelas com DMO diminuída apresentaram menor peso comparada as com DMO normal, sem diferença estatisticamente significativa. Porém, sabe-se que peso corporal exerce forte influência na remodelação óssea, dessa forma o grupo com menor peso pode não ter sofrido estímulo suficiente em termo de intensidade de forças de reação do solo. Este fato pode explicar, em parte, os resultados obtidos com o pedômetro neste estudo. Outra possível explicação seria que a caminhada é atividade natural, segura, acessível e amplamente recomendada por diferentes órgãos de saúde. Tal fato pode ter afetado o comportamento das mulheres que já tinham diagnóstico de baixa DMO.

Observou-se que 80\% das mulheres no grupo de DMO diminuída não realizavam suplementação com cálcio e 70\% não utilizavam vitamina $D$, combinada a baixa ingestão diária de ambos. Assim, a região lombar poderia estar apresentando perda óssea ao invés de ganho no dia a dia daquelas que apresentaram elevado número de passos diários. Foi constatada perda óssea, de forma semelhante, em atletas jovens em atividades de endurance de alto rendimento consequente da amenorreia secundária² . Porém, essa baixa DMO presente na ausência de hormônios, talvez possa ser corrigida aumentando-se carga dos exercícios e diminuindo frequência ou repetições; caso de ginastas olímpicas oligoamenorreicas que apresentam maior DMO que mulheres sedentárias².

Sobre o histórico de sobrecarga óssea, neste estudo houve risco de cinco e meio vezes maior de mulheres que não praticaram atividade física, de sobrecarga óssea de moderada a alta na fase de adolescência até adulta jovem, de apresentarem diminuição da DMO em comparação às com DMO normal. Para isso também é de fundamental importância nutrição adequada 21,28 e quanto maior o pico de massa óssea na fase adulta jovem, mais protegida contra osteoporose estará nas idades mais avançadas ${ }^{29}$. Corroborando conosco, Daly e Bass $^{30}$ relataram que exercícios com sobrecarga óssea são importantes ao longo de toda vida inclusive na velhice.

Uma limitação desse estudo foi a não identificação da dominância dos membros o que pode ter sido fator de confusão na análise da força muscular. 


\section{CONCLUSÃO}

Este estudo demonstrou que testes de força e nível de atividade física atual não foram sensíveis o suficiente para detectar alterações na DMO nas mulheres estudadas. Porém, torna-se relevante identificar os fatores de risco para prevenção da osteoporose, sendo que, testes de fácil aplicação, amplamente utilizados no dia a dia, podem servir como método de triagem de indivíduos em risco de apresentar baixa $\mathrm{DMO}$, quando associados a outros fatores de risco. Nesta direção os resultados deste estudo, apresentando risco aumentado em cerca de seis vezes nas mulheres que não praticaram atividade física de sobrecarga óssea de moderada à alta na fase da adolescência até a idade adulta, de apresentarem diminuição da DMO em comparação às mulheres com DMO normal, consolidam a importância da atividade física.

Todos os autores declararam não haver qualquer potencial conflito de interesses referente a este artigo.

\section{REFERÊNCIAS}

1. Sievänen $\mathrm{H}$, Kannus $\mathrm{P}$, Nieminen V, Heinonen A, Oja P, Vuori I. Estimation of various mechanical characteristics of human bones using dual energy $\mathrm{x}$-ray absorptiometry: methodology and precision. Bone. 1996;18(1):175-27S

2. Cadore EL, Brentano MA, Kruel LFM. Efeitos da atividade física na densidade mineralóssea e na remodelação do tecido ósseo. Rev Bras Med Esporte. 2005;11(6):373-9.

3. Kemmler W, Weineck J, Kalender WA, Engelke K. The effect of habitual physical activity, non-athletic exercise, muscle strength, and $\mathrm{VO} 2$ max on bone mineral density is rather low in early postmenopausal osteopenic women. J Musculoskelet Neuronal Interact. 2004;4(3):325-34.

4. Gába A, Kapuš O, Pelclová J, Riegerová J. The relationship between accelerometer-determined physical activity (PA) and body composition and bone mineral density (BMD) in postmenopausal women. Arch Gerontol Geriatr. 2012; 54(3):315-21

5. Shackelford LC, LeBlanc AD, Driscoll TB, Evans HJ, Rianon NJ, Smith SM, et al. Resistance exercise as a countermeasure to disuse-induced bone loss. J Appl Physiol. 2004;97(1):119-29.

6. Anjos EM, Cunha MR, Ribas DIR, Gruber CR. Avaliação da performance muscular de idosas nãosedentárias antes e após aplicação de um programa de exercícios de equilíbrio. Evaluation of muscular performance in not sedentary elderly before and after the application of an exercise program for balance. Rev Bras Geriatr Gerontol. 2012;15(3):459-67.

7. Costa EL, Bastos PSC, MouraMS, SousaTS, Lemos A, Pedrosa MAC. Efeitos de um programa de exercícios em grupo sobre a força de preensão manual em idosas com baixa massa óssea. Arq Bras Endocrinol Metab. 2012;56(5):313-8.

8. Aoyagi Y, Park H, Park S, Yoshiuchi K, Kikuchi H, Kawakami H, et al. Interactive effects of milk basic protein supplements and habitual physical activity on bone health in older women: a 1-year randomized controlled trial. Int Dairy J. 2010;20(10):724-30.

9. Sun W, Watanabe M, Tanimoto Y, Kono R, Saito M, Hirota C, et al. Assessment of the best gait parameter in relation to bone status in community-dwelling young-old and old-old women in Japan. Arch Gerontol Geriatr. 2009; 49(1):158-61.

10. Thompson JL, Landry AS, Zoellner JM, Tudor-Locke C, Webster M, Connell C, et al. Several steps/ day indicators predict changes in anthropometric outcomes: HUB City Steps. BMC Public Health. 2012;12:983: 1-11

11. Tudor-Locke C, Bassett DR Jr. How many steps/day are enough? Preliminary pedometer indices for public health. Sports Med. 2004;34(1):1-8.

12. Thompson DL, Rakow J, Perdue SM. Relationship between Accumulated Walking and Body Composition in Middle-Aged Women. Med Sci Sport Exer. 2004;36 (5):911-14.

13. Boyer KA, Kiratli BJ, Andriacchi TP, Beaupre GS. Maintaining femoral bone density in adults: how many steps per day are enough? Osteoporos Int. 2011;22(12):2981-8.

14. Fisberg RM, Marchione DML, Colucci ACA. Avaliação do consumo alimentar e da ingestão de nutrientes na prática clínica.Assessment of food consumption and nutrient intake in clinical practice.Arq Bras Endocrinol Metab. 2009;53(5):617-24.
15. Dolan SH, Williams DP, Ainsworth BE, Shaw JM. Development and reproducibility of the bone loading history questionnaire. Med Sci Sport Exer. 2006;38 (6):1121-31.

16. Novaes RD, Miranda AS, SilvaJO, Tavares BVF, Dourado VZ. Equações de referência para a predição da força de preensão manual em brasileiros de meia idade e idosos. Fisioter Pesq. 2009;16(3):217-22.

17. Neto LSS, Karnikowiski MGO, Tavares AB, Lima RM. Associação entre sarcopenia, obesidade sarcopênica e força muscular com variáveis relacionadas de qualidade de vida em idosas. Association between sarcopenia, sarcopenic obesity, muscle strength and quality of life variables in elderly women. Rev Bras Fisioter. 2012;16(5):360-7.

18. Pinto LG, Dias RMR, Salvador EP, Figueira Júnior A, Lima CVG. Efeito da utilização de bandas elásticas durante aulas de hidroginástica na força muscular de mulheres. Rev Bras Med Esporte. 2008;14(5):450-3.

19. Tudor-Locke CA, Ainsworth BE, Whitt MC, Thompson RW, Addy CL, Jones DA. The relationship between pedometer-determined ambulatory activity and body composition variables. Int J Obes Relat Metab Disord. 2001;25 (11):1571-8.

20. Tudor-Locker C, Ham SA, Macera CA, Ainsworth BE, Kirtland KA, Reis JP, et al. Descriptive epidemiology of pedometer-determined physical activity. Med Sci Sports Exer. 2004;36(9):1567-73.

21. Pinto Neto AM, Soares A, Urbanetz A, Souza ACA, Ferrari AEM, Amaral B, et al. Consenso Brasileiro de Osteoporose 2002. Rev Bras Reumatol. 2002;42(6):343-54.

22. Alexandraki KI, Syriou V, Ziakas PD, Apostolopoulos NV, Alexandrakis Al, Piperi C, et al. The knowledge of osteoporosis risk factors in a Greek female population- Maturitas. 2008;59(1):38-45.

23. Minnock E, Cook R, Collins D, Tucker J, Zioupos P. Using risk factors and quantitative ultrasound to identify postmenopausal caucasian women at risk of osteoporosis. J Clin Densitom. 2008;11(4):485-93.

24. Marin RV, Pedrosa MA, Moreira-Pfrimer LD, Matsudo SM, Lazaretti-Castro M. Association between lean mass and handgrip strength with bone mineral density in physically active postmenopausal women. J Clin Densitom. 2010;13(1):96-101.

25. Kato T, Terashima T, Yamashita T, Hatanaka Y, Honda A, Umemura Y. Effect of low-repetition jump training on bone mineral densityin young women. J Appl Physiol. 2006;100(3):839-43.

26. Torstveit MK, Sundgot-Borgen J. Low bone mineral density is two to three times moreprevalent in non-athletic premenopausal women than in elite athletes: a comprehensive controlled study. $\mathrm{Br} J$ Sports Med. 2005;39 (5):282-7

27. Shibata Y, Ohsawa I, Watanabe T, Miura T, Sato Y.Effects of physical training on bone mineral density and bone metabolism. J Physiol Anthropol Appl Human Sci. 2003;22 (4):203-8.

28. Devine A, Dhaliwal SS, Dick IM, Bollerslev J, Prince RL. Physical activity and calcium consumption are important determinants of lower limb bone mass in older women. J Bone Miner Res. 2004;19(10):1634-9.

29. Turner CH, Robling AG. Designing exercise regimens to increase bone strength. Exerc Sport Sci Rev. 2003;31(1):45-50.

30. Daly RM, Bass SL. Lifetime sport and leisure activity participation is associated with greater bone size, quality and strength in older men. Osteoporos Int. 2006;17(8):1258-67. 